\title{
Avaliação da Assistência Farmacêutica na atenção primária no município de Petrolina (PE)
}

\author{
Pharmaceutical Service evaluation in primary health care from the city of \\ Petrolina, Pernambuco, Brazil
}

Eugênio Bispo da Silva Júnior', Luciana Macatrão Nogueira Nunes ${ }^{1}$

\begin{abstract}
RESUMO
Introdução: A Assistência Farmacêutica (AF) representa hoje um dos setores de maior impacto financeiro no âmbito das Secretarias de Saúde e a tendência de demanda por medicamentos é crescente. A ausência de um gerenciamento efetivo pode acarretar grandes desperdícios de recursos escassos. Objetivo: Avaliar a AF em Petrolina, descrevendo as características das etapas do ciclo executadas pelo município. Método: Estudo observacional, longitudinal e descritivo com abordagem qualiquantitativa, desenvolvido por meio da aplicação de questionários semiestruturados aos voluntários de 15 Unidades de Saúde da Família (USFs). Resultados: Observou-se que $80 \%$ das USFs estudadas realizam a etapa de programação do ciclo da AF. No entanto, não há atualização de fichas de prateleiras em 20\% delas e apenas $47 \%$ realizam o acompanhamento de estoque. No quesito armazenamento dos medicamentos foram revelados problemas quanto à infiltração/umidade. A dispensação deles, na maioria das vezes, é realizada por auxiliares de enfermagem nas USFs, não existindo capacitação periódica desses profissionais. Conclusão: Foi constatado que a AF na Atenção Primária em Petrolina enfrenta dificuldades relacionadas à falta de apoio estrutural para o trabalho, além da falta de medicamentos e capacitação incipiente dos profissionais. Estes problemas são considerados passíveis de serem minimizados ou superados a partir da adoção da prática de reorientação da AF incentivada pela Política Nacional de Medicamentos.
\end{abstract}

Palavras-chave: assistência farmacêutica; política nacional de medicamentos; atenção primária à saúde.

\begin{abstract}
Introduction: The Pharmaceutical Service (PS) currently represents one of the sectors with greatest financial impact in the Departments of Health and the trend on demand for drugs is growing. The absence of effective management can lead to a big waste of scarce resources. Objective: To evaluate the PS in the city of Petrolina, Brazil, describing the characteristics of the cycle steps performed by the municipality. Method: Longitudinal and descriptive study with qualitative and quantitative approach, developed by applying semi-structured questionnaires to volunteers from 15 of the Family Health Facilities (FHF). Results: It was shown that $80 \%$ of the studied USF perform the forecasting stage of the cycle of PS. However, there is no update on the shelve sheets in $20 \%$ of FHF and only $47 \%$ use the tracking stock. On the issue of storage of medicines, problems were revealed about the infiltration/moisture. The dispensing of drugs, most often, is performed by nursing assistants in FHF, with no periodic training of these professionals. Conclusion: It was detected that PS in Primary Health Care in Petrolina faces some difficulties related to a lack of structural support for the work, besides a lack of drugs and training of the emerging professionals. These problems are considered likely to be minimized or overcome with the adoption of practices encouraged by the reorientation of the PS National Drug Policy.
\end{abstract}

Keywords: pharmaceutical services; national drug policy; primary health care. 


\section{INTRODUÇÃO}

Com o propósito de garantir o acesso da população aos medicamentos considerados essenciais, bem como a necessária segurança, eficácia e qualidade destes e a promoção do uso racional, o Ministério da Saúde aprovou a Política Nacional de Medicamentos (PNM), por meio da publicação da Portaria $\mathrm{n}^{\circ} 3.916$ em 30 de outubro de 1998, que efetivou a Assistência Farmacêutica (AF) no Sistema Único de Saúde (SUS) ${ }^{1}$.

Por seu caráter sistêmico, multiprofissional e não pontual, a AF não se restringe aos procedimentos de abastecimento de medicamentos, como há décadas vem sendo entendido. Sua organização implica em promover a necessária articulação dos vários procedimentos ou etapas, de modo a assegurar a oferta de medicamentos, em qualquer nível de atenção à saúde, segundo critérios de necessidade, quantidade, qualidade, risco/benefício, custo/benefício, entre outros, visando a utilização racional dos medicamentos na atenção à saúde ${ }^{2,3}$.

A reorientação da AF está fundamentada na descentralização da gestão, na promoção do uso racional dos medicamentos, na otimização e eficácia do sistema de distribuição no setor público e no desenvolvimento de iniciativas que possibilitem a redução nos preços dos produtos ${ }^{4,5}$. Sua organização procura superar a fragmentação da área, valorizando a articulação entre as diversas partes que compõem o seu próprio sistema de saúde, estabelecendo fluxos na construção de um conjunto articulado que influencia e é influenciado por cada um de seus componentes ${ }^{6}$.

Os conhecimentos técnicos e científicos para o desenvolvimento organizacional da AF são fundamentais para uma perfeita condução da estratégia proposta, dando uma sequência lógica ao processo. Para sua efetiva implementação, é fundamental ter como princípio básico norteador o Ciclo da Assistência Farmacêutica, constituído pelas etapas de seleção, programação, aquisição, armazenamento, distribuição, dispensação de medicamentos, com suas interfaces nas ações de atenção à saúde ${ }^{7,8}$. No Ciclo da Assistência Farmacêutica, o resultado de uma atividade é ponto de partida para outra e a ausência ou a execução de forma inadequada de uma delas acaba impedindo o correto funcionamento de todo o ciclo. Tal fato pode resultar na desorganização dos serviços, comprometendo o objetivo dos programas ${ }^{9}$. Dessa forma, a AF traz consigo enormes dificuldades e desafios, tanto no que se refere à sua estruturação quanto no que diz respeito à ampliação do acesso da população a medicamentos. Para saná-los, é de suma importância a organização dos mesmos pelos gestores estaduais e municipais, no sentido de buscar sempre o melhor a fim de um funcionamento eficiente.

A partir desse contexto, e junto à escassez de estudos que abordam o tema proposto em nível municipal e, principalmente, na região do sub-médio do São Francisco, foi idealizada esta pesquisa, cujo objetivo é avaliar a Assistência Farmacêutica na Atenção Básica em Petrolina, município situado no sertão pernambucano, na perspectiva de contribuir com uma análise crítica dessa organização no SUS, imprescindível para o conhecimento da situação da saúde pública local e o comprometimento dos gestores públicos com a população.

\section{MÉTODOS}

Localizada na mesorregião São Francisco, Petrolina ocupa a área de $4.737,1 \mathrm{~km}^{2}$ e representa $4,81 \%$ do estado de Pernambuco, com população total de 281.851 habitantes, sendo 166.279 $(76,1 \%)$ deles moradores da zona urbana e 52.259 (23,9\%) da zona rural (IBGE, 2000) ${ }^{10}$. O Índice de Desenvolvimento Humano Municipal (IDH-M) é 0,748, o que correspondia, no período, à $6^{a}$ posição no ranking estadual e em $1.948^{\mathrm{a}}$ no nacional. A rede de saúde local é composta por três hospitais, 333 leitos, 49 ambulatórios e 175 agentes comunitários de saúde pública. E a taxa de mortalidade infantil, segundo dados do Departamento de Informática do Sistema Único de Saúde (DATASUS) é de 59,72 para cada mil nascidos vivos.

O estudo delineado foi do tipo observacional, longitudinal e descritivo, com abordagem qualiquantitativa da Assistência Farmacêutica na rede básica do SUS no município de Petrolina.

A pesquisa foi desenvolvida em 15 Unidades de Saúde da Família (USFs), das quais cinco funcionaram como sede operacional dos Núcleos de Apoio à Saúde da Família (NASFs) entre os meses de maio a julho de 2010. Os critérios de inclusão da unidade de saúde na pesquisa se basearam no atendimento de algumas exigências, como: possuir farmácia para dispensação de medicamentos; pertencer à gestão do município e não estar localizada em zona rural. Os funcionários entrevistados nas unidades deveriam ter vínculo empregatício de um período mínimo de três meses de exercício junto à secretaria municipal de saúde de Petrolina, além de atuar em uma das unidades selecionadas para a pesquisa.

De acordo com $\mathrm{Gil}^{11}$, o questionário é o meio mais rápido, barato e prático de obtenção de informações, além de garantir o anonimato ${ }^{11}$. Dessa forma, o instrumento escolhido para a coleta de dados foi um questionário semiestruturado, elaborado de acordo com a Instrução Normativa para Organização da AF do Ministério da Saúde ${ }^{12}$, subdividido em duas partes e contendo um total de 211 perguntas. A primeira parte foi aplicada aos farmacêuticos vinculados aos NASFs com o propósito de coletar dados relativos a esse âmbito e suas respectivas unidades. A segunda englobou perguntas específicas à coordenação da assistência farmacêutica, ficando restrita apenas ao coordenador. $\mathrm{O}$ questionário abordou questões variadas relacionadas à organização, gerência e ao funcionamento pertinentes a todas as etapas do ciclo da AF, como por exemplo: estrutura física externa e interna, qualidade do armazenamento de medicamentos, aquisição e dispensação. Os indicadores de qualidade das etapas (estrutura e processo) foram estabelecidos a partir das sugestões contidas nas instruções técnicas para organização da $\mathrm{AF}^{12,13}$ e utilizadas como parâmetro de diferença entre a situação desejada e a atual. 
As entrevistas, previamente agendadas, ocorreram após a autorização da Coordenação da Assistência Farmacêutica da Secretaria Municipal de Saúde de Petrolina (PE) manifestada por uma Carta de Anuência. Os entrevistados foram informados sobre os objetivos da pesquisa e assegurados com relação ao sigilo e preservação do anonimato. Todos assinaram o Termo de Consentimento Livre e Esclarecido (TCLE) elaborado pelo pesquisador de acordo com a Resolução 196/96 e o projeto foi aprovado pelo Comitê de Ética e Pesquisa em Seres Humanos da Universidade Federal do Vale do São Francisco (UNIVASF; protocolo CAAE no 0014.0.441.000-11).

Assim como todas as pesquisas que adotam o questionário como instrumento de coleta de dados, há sempre limitações com relação à sua confiabilidade. No entanto, havendo treinamento prévio e explicação dos objetivos da pesquisa aos entrevistados, esses problemas puderam ser minimizados.

A interpretação dos dados foi baseada na análise do fenômeno ao longo do seu desenvolvimento, e os resultados agrupados em percentuais e dispostos em tabelas e gráficos utilizando-se os programas Excel 2010 e Epiinfo 3.5.1.

\section{RESULTADOS E DISCUSSÃO}

A Secretaria Municipal de Saúde de Petrolina possui em seu organograma a Coordenação de Assistência Farmacêutica. A equipe da $\mathrm{AF}$ do município dispõe somente de seis farmacêuticos, sendo cinco vinculados ao NASF, dando suporte, cada um, a oito USFs vinculadas a uma unidade sede. A Secretaria também conta com um farmacêutico coordenador, vinculado à Coordenação da AF.

Com relação ao perfil dos pesquisados, quatro eram do sexo feminino e três do masculino. Todos concluíram a graduação em Ciências Farmacêuticas e o tempo de trabalho variava de um ano a seis meses, com jornada de trabalho de 40 horas.

O setor de compras realiza licitações anuais para aquisição dos medicamentos. O mesmo utiliza o registro técnico dos fornecedores e parecer técnico dos produtos quando a coordenação da Assistência Farmacêutica Municipal solicitava a aquisição de medicamentos. Dessa forma, a escolha de licitações para compras de medicamentos feitas pelo município atende aos princípios de legalidade e transparência da administração pública.

No estudo realizado por Vieira ${ }^{14}$, em que foram avaliados 660 municípios do Brasil pela Corregedoria Geral da União (CGU), notou-se que 19\% deles não observaram as normas vigentes para a realização de compras públicas. Embora essas normas tornem o processo de aquisição de medicamentos ou de qualquer outro bem e serviço mais lento, o seu objetivo principal é conferir economia e transparência aos atos administrativos relacionados. O percentual encontrado indica que podem estar havendo perdas devido à inobservância das normas, à ausência de qualificação e ao treinamento do pessoal responsável por sua execução.
Quando questionado a respeito da existência da Comissão de Farmácia Terapêutica (CFT), foi respondido que não existia de forma regulamentada tal comissão. A mesma exerce um papel fundamental em relação ao apoio das atividades de seleção, exigindo a participação de outros profissionais, além do farmacêutico, na inclusão ou exclusão de medicamentos do componente básico. Observou-se também que a $\mathrm{AF}$ não adotava, ainda, a Relação Municipal de Medicamentos Essenciais (REMUME). A seleção é feita pelos farmacêuticos que compõem a equipe da AF, seguindo a Relação Nacional de Medicamentos Essenciais (RENAME), uma das diretrizes da Política Nacional de Medicamentos.

A maioria das USFs (80\%) admite realizar a etapa de programação do ciclo da AF. No entanto, não há atualização de fichas de prateleiras em $20 \%$ delas, e apenas $47 \%$ realizam o acompanhamento de estoque (Figura 1). No estudo de Vieira $^{14}$, constatou-se que $71 \%$ dos municípios apresentaram controle de estoque ausente ou deficiente, refletindo falha grave de gestão.

Este controle poderia ser realizado de várias formas, desde as mais simples, como o acompanhamento das fichas de prateleira, até a utilização de um sistema informatizado, dependendo dos recursos disponíveis. O mais importante é que, qualquer que seja a forma adotada, a informação seja obrigatoriamente segura e confiável, pois sem um sistema de informação eficiente, dificilmente se terá condição para realizar um bom gerenciamento de estoque.

A Assistência Farmacêutica Municipal não apresenta Central de Abastecimento Farmacêutico, definida como uma unidade que tem por finalidade manter e abastecer de modo dinâmico os estoques de medicamentos com qualidade e quantidades adequadas e como um almoxarifado que armazena e distribui todos os produtos e equipamentos de saúde do município, incluídas as USFs. Relatou-se que essa distribuição de medicamentos é realizada mensalmente, o que representa uma frequência elevada considerando o reduzido número de funcionários lotados no almoxarifado.

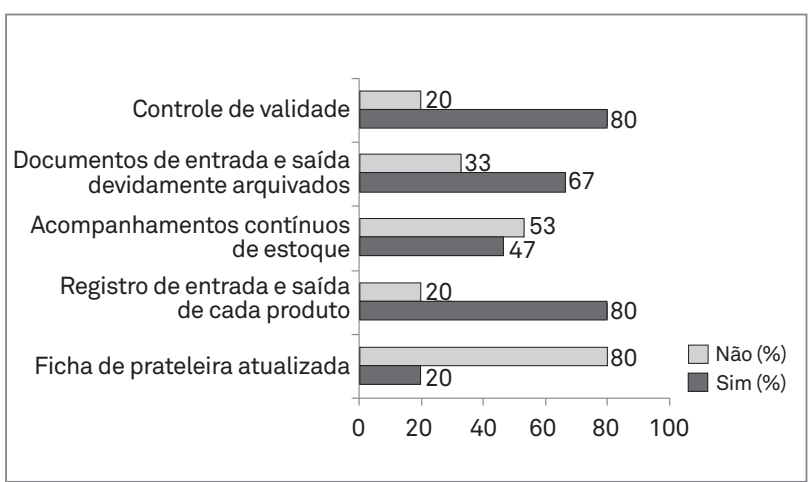

Figura 1: Distribuição das Unidades de Saúde da Família que realizam a etapa de programação do ciclo da Assistência Farmacêutica relacionado ao controle de estoque 
No quesito armazenamento dos medicamentos, foram revelados problemas quanto à infiltração/umidade: $27 \%$ das unidades apresentaram situação desfavorável. O procedimento de registro de temperatura de geladeira para produtos termolábeis foi constatado em apenas $13 \%$ das USFs e não houve relato a respeito do controle de temperatura ambiente (Figura 2).

Verifica-se também que todos os medicamentos estavam dispostos em estantes ou prateleiras. Sabe-se que em muitas USFs os medicamentos são armazenados e dispensados nos consultórios, não havendo um local específico para eles, o que dificulta o controle de estoque. Nota-se que não há apoio da maior parte dos profissionais gestores para reverter essa situação. Em algumas unidades com condições extremamente precárias, é disponível uma sala específica para a farmácia, na qual há o armazenamento e a dispensação. Vale salientar que uma armazenagem racional aperfeiçoa recursos e assegura a qualidade dos medicamentos. O problema de condições de armazenamento não é único em Petrolina. Na pesquisa de $V_{\text {Vieira }}{ }^{15}$, foi constatado que $47 \%$ dos municípios estudados estavam sujeitos a condições inadequadas de armazenamento ${ }^{14}$.

Com relação à dispensação nas farmácias, a prática é realizada, geralmente, por um auxiliar de enfermagem nas USFs quando o farmacêutico não se encontra na unidade, não existindo capacitação periódica desses profissionais. A respeito dos Procedimentos Operacionais Padrão (POPs), apenas 20\% das USFs responderam que o possuíam para normatização de procedimentos de dispensação. No referido estudo observou-se que a maioria (93\%) das USFs realiza a dispensação de medicamentos mediante a apresentação da prescrição médica. A unidade remanescente dispensa medicamentos mediante prescrições de outros profissionais de saúde, porém é necessário avaliar minuciosamente os aspectos legais com relação a essa prática.

A dispensação pela prescrição médica não contribui somente para o uso racional de medicamentos, como também auxilia, sendo um indicador da AF em relação ao desenvolvimento do planejamento e controle de estoque, uma vez que o volume de prescrições de um determinado medicamento ou esquema terapêutico pode ajudar a definir o cálculo da aquisição pelo mês de consumo.

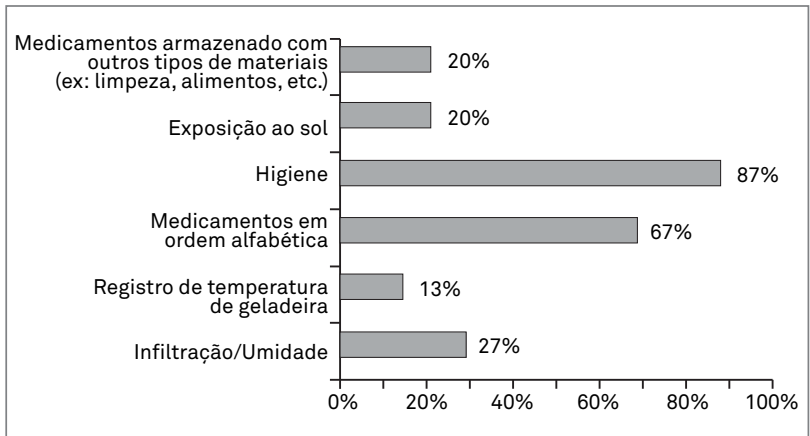

Figura 2: Constatação das condições de armazenamento de medicamentos nas Unidades de Saúde da Família
É válido observar que a falta de remédio e a capacitação insuficiente foram citados por todos os farmacêuticos como alguns dos principais problemas apresentados nas USFs. O primeiro pode, dentre outras razões, ser devido a uma seleção inapropriada. De acordo com o Ministério da Saúde ${ }^{6}$, o ponto de partida dos fármacos no ciclo da assistência farmacêutica é a seleção, sendo fundamental a adoção de critérios eficazes e seguros para o atendimento da população a partir do diagnóstico prévio da área de atuação, como as doenças mais prevalentes, a fim de garantir a medicação essencial para o tratamento dos possíveis pacientes daquela determinada área.

Vale salientar que o espaço físico e infraestrutura (acabamento em geral, infiltrações, condições de pintura, etc) também foram colocados como um dos principais problemas para a implementação da AF das USFs. Em relação ao espaço físico, $67 \%$ tem área exclusiva para dispensação de medicamentos, mas em $40 \%$ ela é insuficiente para tal prática, restando um ambiente em desconformidade com as diretrizes para a estruturação de farmácias básicas no âmbito do SUS.

Em se tratando de ambiente interno, apenas 33\% das edificações foram reconhecidas como em bom estado. Já em relação ao externo, a pesquisa indica que $80 \%$ sofrem com poluição ou contaminação. Em apenas $7 \%$ existia bloqueio de acesso de animais e em $40 \%$ foi relatado esgoto a céu aberto. A coleta de lixo regular é apresentada em 75\% das unidades estudadas e em nenhuma foi encontrada proteção contra insetos e roedores (como janelas teladas e dedetização periódicas). Conforme Araújo e Freitas ${ }^{8}$, a atividade da AF nas Unidades de Saúde da Família torna-se praticamente impossível, pois na farmácia deságuam quase todas as mazelas do sistema de saúde, por ela se enquadrar no elo final do processo de atendimento ${ }^{15}$.

Manifestamos a importância deste estudo no sentido de gerar uma reflexão que permita compreender a significância da gestão da AF na sua prática, no seu contexto e no cotidiano nos serviços de saúde do município de Petrolina. A execução da pesquisa de campo nas USFs possibilitou aprender determinados elementos da AF local por meio de um olhar mais próximo da realidade, não centrado apenas nas questões operacionais, mas na visão dos farmacêuticos que fazem cobertura desse campo.

Nas condições da pesquisa pode-se constatar também que a AF na atenção primária em Petrolina esbarra em dificuldades relacionadas à falta de apoio estrutural para o trabalho, talvez como um reflexo da ausência de profissionais farmacêuticos ocupando cargos estratégicos na SMS. Notou-se também insuficiência no número de profissionais, os sobrecarregando de maneira sobre-humana e responsabilizando pelas falhas resultantes do acúmulo de atribuições, uma vez que é escasso o treinamento e/ou capacitação de outros profissionais envolvidos no ciclo do medicamento nas unidades e almoxarifado. 


\section{REFERÊNCIAS}

1. Brasil. Ministério da Saúde. Portaria n 3.916/GM, de 30 de outubro de 1998. Política Nacional de Medicamentos. Brasília: Diário Oficial da União;1998.

2. Dupim JAA. Assistência farmacêutica: um modelo de organização. Belo Horizonte: Segrac; 1999.

3. De Bernardi CLB, Bieberbach EW, Thomé HI. Avaliação da assistência farmacêutica básica nos municípios de abrangência da $17^{\circ}$ Coordenadoria Regional de Saúde do Rio Grande do Sul. Saude Soc. 2006;15(1):73-83.

4. Brasil. Ministério da Saúde. Portaria n 3.916, de 30 de outubro de 1998. Aprova a política nacional de medicamentos. Diário Oficial [da] República Federativa do Brasil, Poder Executivo, Brasília, DF: 10 nov. 1998; Seção 1, p.18.

5. Brasil. Conselho Nacional de Secretários de Saúde - CONASS. Legislação do SUS. Brasília: CONASS; 2003.

6. Marin N, Luiza VL, Osorio-de-Castro CGS. Assistência farmacêutica para gerentes municipais. Rio de Janeiro: OPAS/OMS; 2003.

7. Vieira FS. Qualificação dos serviços farmacêuticos no Brasil: aspectos inconclusos da agenda do Sistema Único de Saúde. Rev Panam Salud Pública. 2008:24(2):91-100.

8. Araújo ALA, Freitas O. Concepcões do profissional farmacêutico sobre a assistência farmacêutica na unidade básica de saúde: dificuldades e elementos para a mudança. Rev Bras Ciênc Farm. 2006;42(1):137-46.

9. Brasil. Ministério da Saúde. Secretaria de Políticas de Saúde. Assistência Farmacêutica: instruções técnicas para sua organização. Brasília: Ministério da Saúde; 2001.
10. Instituto Brasileiro de Geografia e Estatística (IBGE). Contagem da populacão 2007. Rio de Janeiro: IBGE; 2007. Disponivel em: <http:// www.ibge.gov.br/home/estatistica/populacao/contagem2009/ popmunic20079 layoutTCU14112007.xls>. Acesso em: maio 2010.

11. Gil AC. Como elaborar projetos de pesquisa. São Paulo: Atlas; 2002.

12. Brasil. Ministério da Saúde. Assistência farmacêutica na atenção básica: instruções técnicas para a sua organização. Brasília: 2002.

13. Brasil. Ministério da Saúde. $11^{\text {a }}$ Conferencia Nacional de Saúde: - Brasil falando como quer ser tratado: efetivando o SUS, acesso, qualidade e humanizacão na atenção à saúde com controle social: relatório final. Brasília: Ministério da Saúde; 2001.

14. Vieira FS. Assistência farmacêutica no sistema público de saúde no Brasil. Rev Panam Salud Pública. 2010;27(2):149-56.

15. Vieira FS. Possibilidades de contribuição do farmacêutico para a promoção da saúde. Ciênc Saúde Coletiva. 2007;12(1):213-20.

\section{Endereço para correspondência}

Luciana Macatrão Nogueira Nunes - Rua da Glória, 214, apto. 1.301 CEP: 20241-180 - Rio de Janeiro (RJ), Brasil -

E-mail: lucianamacatrao2@yahoo.com.br

Fonte de financiamento: Programa de Educação pelo Trabalho para a Saúde (PET-SAÚDE), do Ministério da Saúde.

Conflito de interesse: nada a declarar 